\title{
The Relationship Between Internal Encoding Style and Obsessive-Compulsive Symptoms in a Subclinical Sample
}

\author{
Sanaâ Belayachi' and Martial Van der Linden ${ }^{1,2}$ \\ 1 Cognitive Psychopathology Unit, University of Liège, Belgium \\ ${ }^{2}$ Cognitive Psychopathology and Neuropsychology Unit, University of Geneva, Switzerland
}

\begin{abstract}
This study examined the relationships between obsessive-compulsive symptoms (OCs) and encoding style in a subclinical sample, by using a questionnaire that evaluates the extent to which preexisting schemata (versus cues from the outside world) affect encoding processes (Lewicki, 2005). Research on encoding style has revealed on one hand the existence of individual differences in the tendency to impose interpretive schemata in the process of encoding, and on the other hand the fact that an extremely internal mode of encoding has been found to be related to an increased propensity to self-perpetuate preexisting schemata. Furthermore, internal encoding may contribute to the development of psychopathological symptoms, through the self-perpetuation of dysfunctional schemata. The results confirmed that OCs are connected with an internal encoding style; specific relationships between obsessive-compulsive disorder (OCD) dimensions and internal encoding style were also found. These results are discussed in terms of the role of encoding style in the perpetuation of OCs, and its relationship to the dysfunctional beliefs characterising OCD.
\end{abstract}

Keywords: obsessive-compulsive disorder, internal and external encoding, encoding style, dysfunctional beliefs, self-perpetuation

Obsessive-compulsive disorder (OCD) is characterised by both obsessions (i.e., recurrent unwanted intrusive thoughts or images, which cause distress) and compulsions (i.e., mental or physical acts performed in a repeated or stereotyped way). Cognitive theories of OCD emphasise the importance of dysfunctional beliefs, such as an inflated personal responsibility, an overestimation of threat, the need to control thoughts, and perfectionism, in the occurrence and maintenance of OCD symptoms (e.g., OCCWG, 2005). Those dysfunctional schemata are thought to produce catastrophic misinterpretations of internal (e.g., intrusive thoughts) or external (e.g., car crash in one's surrounding area) stimuli (e.g., Rachman, 1997). Such maladaptive appraisals can induce distress and anxiety, and could, in turn, lead to compulsions. OCD individuals experience compulsions as effective strategies to counter both bad outcomes (i.e., because feared events did not occur) and distress; nevertheless, while obsession-related distress is momentarily attenuated, experience of effectiveness progressively reinforces irrational beliefs, thereby increasing the likelihood

Address for correspondence: Sanaâ Belayachi, Cognitive Psychopathology Unit, Department of Cognitive Sciences, University of Liège, Belgium. E-mail: sanaa.belayachi@ulg.ac.be 
of further compulsive behaviors (e.g., Salkovskis, 1985; Rachman, 2002). In addition to this paradoxical effect of compulsions, interpretive biases may also play an important role in the maintenance and recurrence of OCD features, as they cause ambiguous stimuli to be interpreted as consistent with irrational thoughts (e.g., Rachman, 1997, 2002). From this perspective, OCD can be characterised by a set of self-perpetuating habits of thinking and behaving (Abramowitz \& Deacon, 2005) that can be understood in the context of a basic mechanism of information processing, that is, the style of encoding.

Indeed, some research has revealed the existence of individual differences in how preexisting, internal schemata (versus external cues from the outside world) affect encoding processes. These differences relate to how 'hasty' (or 'internal', i.e., based on internal encoding categories) versus 'conservative' (or 'external', i.e., based on data from external stimuli) the encoding processes are (Lewicki, 2005). According to Lewicki, encoding style can be conceptualised as the relative amount of supportive evidence a perceiver needs to accumulate before imposing an interpretive category on a cue (i.e., the threshold amount necessary to instantiate an interpretive schema). When stimuli are ambiguous, encoding algorithms may nonconsciously impose preexisting interpretative categories on them even if the stimuli objectively do not match those categories very well (e.g., Lewicki, Hill, \& Sasaki, 1989). Research indicates that high levels of internal encoding increases the probability that the environmental cues will be interpreted in terms of preexisting (internal) encoding schemata, thus providing support for those representations and contributing to their reinforcement through the process of 'self-perpetuation' (e.g., Lewicki, 2005; Lewicki, Hill, \& Czyzewska, 1992). Furthermore, such a disposition to impose internal personal schemata could have dysfunctional long-term consequences (i.e., the self-perpetuation of maladaptive schemata) and may even lead to content-related dysfunctions, such as exaggerated emotional reactions to specific categories of cues and psychological symptoms (Hill et al., 1989; Lewicki, 2005; Lewicki, et al., 1992). This vicious circle of gradual increase of attentional/perceptual biases and emotional reaction is considered to play a crucial role in the causation and maintenance of psychopathological disorders.

The objective of the present study was to explore the relationship between internal encoding style and OC symptoms (OCs) in individuals with subclinical levels of OCs (i.e., nonclinical participants). We hypothesised that 'the level of internal encoding' may be related to the level of OCs. We will also examine and compare the different OC dimensions on the measure of encoding style. Indeed, many studies highlight the OCD heterogeneity and the possibility that different subtypes of symptoms may be distinguished according to their cognitive correlates (e.g., Pigott, Myers, \& Williams, 1996; Mataix-Cols et al., 2004). Thus, we will also examine the specific relationships between internal encoding style and the six OCD dimensions. In order to assess the style of encoding, we will use the internal and external encoding style questionnaire (ESQ; Lewicki, 2005). This scale was constructed on the basis of the assumption that the threshold of instantiation of schemata (i.e., the amount of evidence needed before a stimulus can be perceived as a concrete exemplar of that schema) may determine the probability, and, therefore, the frequency of experiencing the commonly observed phenomenon 'split second illusions' (i.e., erroneous impression to recognise something specific, only to realise a moment later that it was something else). Because internal encoders are more likely to more hastily impose, even wrong, internal schemata in the course of 
perception, they should experience split second illusions more frequently when identifying certain known objects or phenomena. A recent French validation study confirmed that encoding style is a continuum extending from extremely internal to extremely external, as scores on the ESQ were found to be normally distributed (Billieux, D'Argembeau, Lewicki, \& Van der Linden, 2009). Internal encoding as measured by the ESQ was found to be related to better recognition performances in tachistoscopic presentations of everyday objects or incomplete displays of letters, and with more self-perpetuation of newly acquired encoding algorithms (see Lewicki, 2005). In addition, high internal encoding scores have been related to psychopathological symptoms such as fantasy proneness, depression, anxiety (Lewicki, 2005), and impulsiveness (Billieux et al., 2009).

\section{Method}

\section{Participants}

Three hundred and twelve undergraduates from the University of Liège participated in the study. Data from two participants were discarded as they were 17 years old at the time of testing. In addition, two participants that appeared to be critical outliers were omitted. The reported results are from the remaining 308 participants (113 males and 195 females $)$, aged between 18 to 36 years old $(M=22$ years; $S D=2.49)$.

\section{Materials}

Obsessive-Compulsive Inventory - Revised (OCI-R, Foa et al., 2002). The validated French version of the OCI-R (Zermatten, Van der Linden, Jermann, \& Ceschi, 2006) is a self-report questionnaire that consists of 18 items evaluating OCD symptoms and is composed of six subscales (each containing three items): Washing, Obsessing, Hoarding, Ordering, Checking and Neutralising. Participants were asked to determine to what extent the situation described in each particular statement had distressed them during the past month, using a 5-point scale from 0 (Not at all) to 4 (Extremely). Total scores range from 0 to 72; the OCI-R checking and washing subscales scores both range from 0 to 12 . The French version of the OCI-R has good overall psychometric properties and a factorial structure that is identical to that observed in Foa et al.'s (2002) original English version (Zermatten et al., 2006).

Encoding Style Questionnaire (ESQ). This scale, developed by Lewicki (2005), is based on the assumption that the threshold of instantiation of schemata should determine the probability, and thus the frequency of experiencing the commonly observed phenomenon of split-second illusions (e.g., erroneously recognising an animal running across the road before finding out a moment later that it was a piece of paper blown by the wind). Indeed, because internal encoders are more likely to more hastily impose encoding schemata (even wrong ones), they should experience split-second illusions more frequently when identifying certain objects or phenomena. This scale consists of 21 items evaluating the level of internal encoding. Only six items (numbers 5, 8, 11, 15, 18, and 21) are diagnostic items; the remaining 15 items are included to disguise the focus of the test. Representative diagnostic items include, 'For a split second from a distance, I sometimes mistake strangers for people I know', and 'Sometimes when I'm driving, I see a piece of paper or a leaf being moved by the wind, and for a split second think that it might be an animal (e.g., a squirrel or a cat)'. Participants respond to each item using a 6-point Likert type 
scale, ranging from 1 (Strongly disagree) to 6 (Strongly agree). Total scores range from 6 to 36; a high score on the ESQ reflects an internal encoding style, whereas a low score reflects an external encoding style. The French version of the ESQ has good overall psychometric properties and a factorial structure that is identical to that observed in the original English version (Billieux et al., 2009).

\section{Procedure}

In a testing session lasting 30 minutes, participants completed the OCI-R and the ESQ in an individual setting, as well as other questionnaires unrelated to the present study.

\section{Results}

Cronbach's alphas indicated good internal consistency for the encoding style (ESQ: .71), and OCD measures (OCI-R total score: .84; Washing: .72; Obsessing: .80; Ordering: .82; Checking: .78; Hoarding: .75), except for the Neutralising subscale that showed a rather low reliability (.59). The sample mean scores for all these measures are shown in Table 1. A statistical test of homogeneity confirmed that the mean score on the ESQ obtained in this study $(M=18.18 ; S D=5.35)$ did not differ from that observed in a sample of 63 undergraduates in the Billieux et al. (2009) study $(p>$.94). Mean scores for OCD dimensions showed that Hoarding and Ordering symptoms were the most frequent, while Neutralising and Washing dimensions were less frequently observed in a subclinical sample, replicating previous studies in which Hoarding and Ordering symptoms were found to be more frequent in the general population than Washing and Neutralising (e.g., Foa et al., 2002; Zermatten et al., 2006). We must point out that, except for the neutralising OCI-R subscale, statistical tests of homogeneity revealed that all OCI-R scores are higher in our study than in a French-speaking sample of 583 undergraduates in the Zermatten et al. (2006) French validation of the OCI-R study ( $p s<.05)$. When compared with OCI-R scores obtained in an English-speaking sample of 477 nonclinical participants in the Foa et al. (2002) original version study, statistical tests of homogeneity revealed that the Washing, Ordering, Neutralising and OCI-R total mean scores from our sample are lower than those reported by Foa et al. (2002) $(p<.05)$; whereas the Checking, Hoarding and Obsessing mean scores were comparable across the two studies (ps > .05).

Pearson correlations were performed in order to explore associations between the measures of OCD and encoding style. These correlations are presented in Table 2 and reveal that, except for the ordering subscale, the measure of encoding style was significantly correlated with all the OCI-R scores (small to medium effects, $r=.18-.35$ ).

In order to investigate the specific relationship between the encoding style and the different OCD dimensions, we performed a regression analysis with the encoding style as a dependent variable and the six OCI-R subscales as independent variables, using the backward exclusion selection procedure. Obsessing $(t=4.17, p<.001, \beta=$ $.23)$, Hoarding $(t=3.68, p<.001, \beta=.20)$, and Washing $(t=3.11, p<.01, \beta=.17)$ emerge as significant independent predictors of internal encoding style, whereas, Checking, Neutralising, and Ordering do not explain a significant proportion of variance of encoding style ( $p s>.26$ ), when controlled for the other OCD dimensions. The checking subscale was the first excluded by the backward elimination selection procedure, followed by the neutralising, and finally by the ordering subscale.

\section{Behaviour Change}




\section{TABLE 1}

Mean Scores and SDs for Encoding Style and OCD Measures

\begin{tabular}{lc}
\hline Dependent variables & Score $(S D)$ \\
\hline Encoding style & $18.18(5.35)$ \\
OCD dimensions & \\
OCl-R Checking & $2.62(2.55)$ \\
OCl-R Washing & $1.58(2.13)$ \\
OCl-R Obsessing & $2.94(2.68)$ \\
OCl-R Hoarding & $4.03(2.77)$ \\
OCl-R Ordering & $3.72(2.91)$ \\
OCl-R Neutralising & $1.03(1.68)$ \\
OCl-R total score & $15.92(9.35)$ \\
\hline
\end{tabular}

Note: $S D$ : standard deviation

\section{TABLE 2}

Pearson Correlations Between OCD Dimensions and Encoding Style Measures

\begin{tabular}{ll}
\hline Encoding style & \\
\hline OCD dimensions & $.18^{*}$ \\
Checking & $.27^{* *}$ \\
Washing & $.32^{* *}$ \\
Obsessing & $.27^{* *}$ \\
Hoarding & .08 \\
Ordering & $.23^{* *}$ \\
Neutralising & $.35^{* *}$ \\
OCl-R total score & \\
\hline
\end{tabular}

Note: ${ }^{*} p<.01,{ }^{* *} p<.001$

\section{Discussion}

The main finding of the present study was that internal encoding style was related to the general OC phenomena as measured by the OCI-R total score. In addition, only the Obsessing, Washing and Hoarding symptoms seemed to have independent relationship with an internal mode of encoding. Overall, our results point to an association between certain OCD symptoms and an overall tendency to impose interpretative schemata on ambiguous stimuli (i.e., internal encoding style). According to the encoding style theory, such an internal mode of encoding in the course of perception leads individuals to have a strong feeling of seeing things (i.e., it is not an hallucination) that are not obviously there (Lewicki, 2005). In other words, individuals with an extreme internal encoding may have an immediate subjective experience similar to that one could undergo if confronted with the actual presentation of the same things; hence, irrational thoughts are experienced as realistic as well and related emotions such as fear and anxiety may reach disproportionate levels.

Furthermore, given that internal encoding may cause encoding biases to be 'indefinitely' strengthened through self-perpetuation, this process may be implicated 
in the gradual amplification of beliefs and related behavioral reactions (i.e., compulsions). For example, if an individual has momentary thoughts about his mother falling sick, which is coincidentally followed by the mother's real hospitalisation, then it may lead to the assumption that those negative thoughts had something to do with the harmful event. Such experiences in people with an extremely internal encoding style could lead to the dysfunctional belief that negative thoughts increase the probability of disastrous events, and thus must be controlled or avoided, which initiates a vicious circle. While in most people, the development of maladaptive thinking may result in slight biases (e.g., superstition) or even in radical changes, extremely internal encoding in OCD individuals may lead to a dysfunctional progression in maladaptive thinking (i.e., strong dysfunctional beliefs). Further studies should be conducted in order to explore the implication of encoding style on the relationship between dysfunctional belief and OCs.

However, we still need to understand the specific cases of Checking, Neutralising and Ordering. Although internal encoding style was correlated, albeit slightly, with Checking and Neutralising, they did not have an independent relationship with encoding style when other OCD symptoms were controlled for. In the case of Checking, the regression analysis revealed that this OCD dimension was the worst predictor of internal encoding scores, as it has been first removed. The rather low implication of dysfunctional beliefs as an underlying motivational factor in the occurrence of checking symptoms in our subclinical sample is one possible explanation of the absence of independent association between those symptoms and internal encoding style. More generally, internal encoding style has the potential for reinforcing dysfunctional beliefs through the process of self-perpetuation. Nevertheless, the studies concerning dysfunctional beliefs in checking symptoms are rather confusing, with some of them observing the importance of dysfunctional beliefs such as harm avoidance (e.g., Tolin, Woods \& Abramowitz, 2003) in checking individuals, while others reported the predominance of another motivational factor, that is, sensory phenomena probably connected with defective action monitoring (i.e., incompleteness, 'not just right experiences', weak sense of task completion or goal satisfaction) in these individuals (e.g., Coles et al., 2003; Tolin, Brady \& Hannan, 2008). Furthermore, a recent study yielded evidence for the motivational heterogeneity of checking symptoms, which predicted both incompleteness and harm avoidance (Ecker \& Gönner, 2008). Thus, work is still needed to clarify this question.

As for neutralising, the absence of a link between neutralising and internal encoding, which is associated with obsessing, is rather inconsistent since the neutralising subscale of the OCI- $\mathrm{R}$ is thought to assess compulsive aspects of obsessions (i.e., thought suppression). However, the psychometric properties of the neutralising subscale may account for this inconsistent result. Indeed, the low alphas, indicative of a lack of homogeneity, shown by this subscale in our study and in others, may be related to the fact that it assesses other OCD dimensions, such as counting-related compulsions (e.g., 'I feel compelled to count while I'm doing things') and superstitions about numbers (e.g., 'I feel there are good and bad numbers'), rather than the neutralisation of unwanted thoughts (Tolin et al., 2008; Zermatten \& Van der Linden, 2008).

Finally, ordering symptoms were not related to internal encoding style at all; on the contrary, there was a slight trend towards significance for the negative association, suggesting that people with ordering proneness may not be related to an internal mode of encoding through which an internal schemata is imposed on stimuli.

\section{Behaviour Change}


Interestingly, it has recently been shown that ordering individuals experience anxiety in obviously disorderly or disorganised environments, but not in ordered surroundings (Radomsky \& Rachman, 2004), confirming that ordering compulsions may constitute behavioral reactions in response to actual environmental modifications (Tallis, 1997), rather than according to specific dysfunctional beliefs that produce anxiety. This is in agreement with previous studies in which the lack of perseverance dimension of impulsivity (i.e., indicative of difficulties inhibiting irrelevant thoughts and remaining concentrated on external stimuli) was found to be positively correlated with obsessing symptoms (Zermatten \& Van der Linden, 2008) but also with internal encoding style (Billieux et al., 2008). On the other hand, it is negatively associated with ordering (Zermatten \& Van der Linden, 2008), supporting the assumption that those symptoms are not triggered by irrelevant threatrelated thoughts (Radomsky \& Rachman, 2004). Thus, our results seem to be consistent with previous studies on encoding style and on OCD.

Before concluding, we should emphasise the fact that the correlational method we used limited the interpretation of the nature of the relationships between encoding style and OCD symptoms. Does an internal encoding style cause the development and maintenance of interpretive biases and consequent irrational strategies in OCD, or is OCD proneness the cause of a chronically internal mode of encoding, through the action of other OCD features such as depression, anxiety, or attentional focus? As we did not control the impact of affective factors such as anxiety and depression in this study, we cannot rule out the possibility that those factors may account for the relationship between the internal mode of encoding and OCD. Thus, the causal direction of the relationship between encoding style and OCD symptoms remains to be explored, as does the potential confounding effect of affective variables.

In summary, the general OCD phenomena and some dimensions of OCD were found to be related to an internal encoding style. In light of the encoding style model, which proposes some underlying mechanisms of cognitive self-perpetuation, our study opens up some new avenues of research on cognitive vulnerabilities in OCD. Nevertheless, although studies on nonclinical samples can generally be extrapolated to clinical samples, further studies should be conducted to replicate our findings in individuals with more severe OCD symptoms (i.e., OCD patients). In addition, while the observed pattern of correlations between encoding style and OCD symptoms appears to be consistent with that presented in the literature, our assumptions are speculative and laboratory experiments are needed to explore the extent to which internal encoding style may lead OCD individuals to be more likely to acquire new and biased encoding schemata and to keep them, even when they appear to be inappropriate. Further work is also needed to see how encoding style interferes with the relationships between dysfunctional beliefs and OCD symptoms.

\section{Acknowledgments}

110 This study was supported by a grant from the French-speaking community of Belgium (Action Recherche Concertée, Convention 06/11-340).

\section{References}

Abramowitz, J.S., \& Deacon, B.J. (2005). Obsessive-compulsive disorder: Essential phenomenology and overlap with other anxiety disorders. In J.S. Abramowitz. \& A.C. Houts (Eds.), Concepts and controversies in obsessive-compulsive disorder (pp. 119-135). New York: Springer. 
Billieux, J., D’Argembeau, A., Lewicki, P., \& Van der Linden, M. (2009). A French adaptation of the internal and external encoding style questionnaire and its relationships with impulsivity. European Review of Applied Psychology, 59, 3-8.

Foa, E.B., Huppert, J.D., Leiberg, S., Langner, R., Kichic, R., Hajcak, G., et al. (2002). The Obsessive-Compulsive Inventory: Development and validation of a short version. Psychological Assessment, 14, 485-496.

Hill, T., Lewicki, P., Czyzewska, M., \& Boss, A. (1989). Self-perpetuating development of encoding biases in person perception. Journal of Personality and Social Psychology, 57, 373-387.

Lewicki, P. (2005). Internal and external encoding style and social motivation. In J.P. Forgas, K.D. Williams, \& S.M. Laham (Eds.), Social motivation: Conscious and unconscious processes (pp. 194-209). New York: Psychology Press.

Lewicki, P., Hill, T., \& Czyzewska, M. (1992). Nonconscious acquisition of information. American Psychologist, 47, 796-801.

Lewicki, P., Hill, T., \& Sasaki, I. (1989). Self-perpetuating development of encoding biases. Journal of Experimental Psychology: General, 118, 323-337.

Mataix-Cols, D., Wooderson, S., Lawrence, N., Brammer, M.J., Speckens, A., \& Phillips, M.L. (2004). Distinct neural correlates of washing, checking, and hoarding symptom dimensions in obsessive-compulsive disorder. Archives of General Psychiatry, 61, 564-576.

Obsessive Compulsive Cognitions Working Group (OCCWG). (2005). Psychometric validation of the Obsessive Beliefs Questionnaire and the Interpretation of Intrusions Inventory: Part 2: Factor analyses and testing of a brief version. Behaviour Research and Therapy, 43, 1527-1542.

Pigott, T.A., Myers, K.R., \& Williams, D.A. (1996). Obsessive-compulsive disorder: A neuropsychiatric perspective. In: R.M. Rapee (Ed.), Current controversies in the anxiety disorders (pp. 134-160). New York: Guiltord.

Rachman, S. (1997). A cognitive theory of obsessions. Behaviour Research and Therapy, 35, 793-802.

Rachman, S. (2002). A cognitive theory of compulsive checking. Behaviour Research and Therapy, 40, 624-639.

Radomsky, A.S., \& Rachman, S. (2004). Symmetry, ordering and arranging compulsive behaviour. Behaviour Research EO Therapy, 42, 893-913.

Salkovskis, P. M. (1985). Obsessional-compulsive problems: A cognitive-behavioural analysis. Behaviour Research and Therapy, 23, 571-583.

Tallis, F. (1997). The neuropsychology of obsessive-compulsive disorder: A review and consideration of clinical implications. British Journal of Clinical Psychology, 36, 3-20.

Tolin, D.F., Brady, R.E., \& Hannan, S. (2008). Obsessional beliefs and symptoms of obsessive-compulsive disorder in a clinical sample. Journal of Psychopathology and Behavioral Assessment, 30, $31-42$.

Tolin, D.F., Woods, C.M., \& Abramowitz, J.S. (2003). Relationship between obsessive beliefs and obsessive-compulsive symptoms. Cognitive Therapy and Research, 27, 657-669.

Zermatten, A., \& Van der Linden, M. (2008). Impulsivity in non-clinical persons with obsessivecompulsive symptoms. Personality and Individual Differences, 44, 1824-1830.

Zermatten, A., Van der Linden, M., Jermann, F., \& Ceschi, G. (2006). Validation of a French version of the Obsessive-Compulsive Inventory - Revised in a nonclinical sample. European Review of Applied Psychology, 56, 151-208.

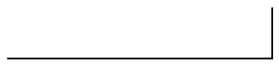

\section{Behaviour Change}

Downloaded from https:/www.cambridge.org/core. University of Basel Library, on 30 May 2017 at 13:48:17, subject to the Cambridge Core terms of use, available at https:/www.cambridge.org/core/terms. https://doi.org/10.1375/bech.27.2.104 